\title{
First crystallographic study of a glutathione transferase from cyanobacteria
}

\author{
E. Mocchetti ${ }^{1}$, A. Hecker², B. Guillot ${ }^{1}$, S. Mathiot ${ }^{1}$, F. Chauvat ${ }^{3}$, C. Cassier-Chauvat ${ }^{3}$, F. Dehez ${ }^{4}$, C. Didierjean ${ }^{1}$ \\ ${ }^{1}$ CRM2, UL, CNRS, Nancy, France \\ ${ }^{2} I A M, U L, I N R A E$, Nancy, France, \\ ${ }^{3}$ I2BC, UPS, CNRS, CEA, Paris-Saclay, France \\ ${ }^{4}$ LPCT, UL, CNRS, Nancy, France \\ eva.mochetti@univ-lorraine.fr, claude.didierjean@univ-lorraine.fr
}

Glutathione transferases (GSTs) are widespread enzymes involved in a number of catalytic and non-catalytic processes [1 and reference herein]. They are mainly known as enzymes of the cellular phase II detoxification system where they catalyse the nucleophilic addition of glutathione (GSH) to a variety of small non-polar compounds. GSTs have been extensively investigated in animals and plants because of their great relevance to human health and agriculture. In contrast, studies in bacteria remain scarce, especially in the cyanobacteria phylum, which encompasses oxygenic photosynthetic prokaryotes with a wide range of morphologies and ecologies. They have key roles in global carbon and nitrogen cycles, contribute strongly to the fixation of atmospheric $\mathrm{CO}_{2}$ and to its storage in ocean sediments (carbon sinks).

The best-studied cyanobacterium Synechocystis PCC6803, which has 6 GSTs, is an attractive organism for deciphering both GST selectivity and redundancy. Preliminary studies show that these GSTs play the expected roles in stress protection. Furthermore, a knockout mutant can be restored by human GST counterparts, demonstrating the conservation of functions throughout evolution [2]. SynGSTC1 is involved in the detoxication of methylglyoxal, a toxic by-product of the cellular metabolism of most organisms [3]. We solved the first crystal structure of a GST from cyanobacteria, namely that of SynGSTC1. It shows the putative active site signature SRAS and belongs to the Chi class of GSTs (Figure 1) and its sequence length is shorter by about 30 residues when compared to the usual GST length ( 220 aa). SynGSTC1 adopts the canonical GST fold that consists of two domains (Figure 1) and exhibits structural similarities with the Ure2p class of GSTs [4]. The structure-function relationships of SynGSTC1 will be presented using innovative tools based on molecular dynamic simulations and charge-density of ultra-high resolution structures.
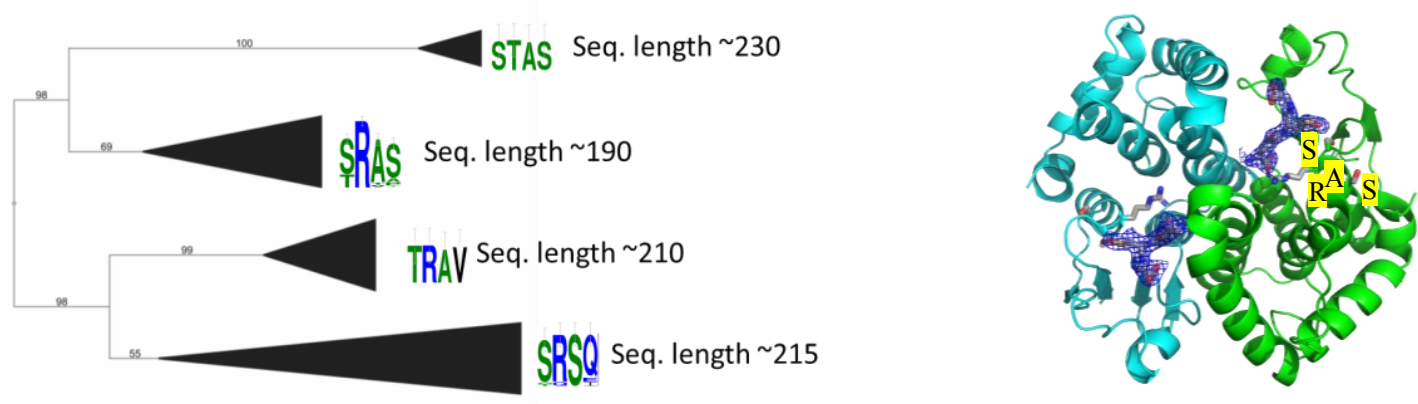

Figure 1. Phylogenetic distribution of SynGSTC1 and its homologs from other cyanobacteria. The putative catalytic signature of each clade is indicated as well as the length of the sequences (left). Crystal structure of SynGSTC1 highlighting the glutathione bound and its electron density. The putative catalytic signature is also shown (right).

[1] Sylvestre-Gonon, E., Law, S. R., Schwartz, M., Robe, K., Keech, O., Didierjean, C., Dubos, C., Rouhier, N. \& Hecker, A. (2019). Frontiers in Plant Science. 10, 1.

[2] Kammerscheit, X., Chauvat, F. \& Cassier-Chauvat, C. (2019). Front. Microbiol. 10, 2248.

[3] Kammerscheit, X., Hecker, A., Rouhier, N., Chauvat, F. \& Cassier-Chauvat, C. (2020). mBio.11, e00882.

[4] Roret, T., Thuillier, A., Favier, F., Gelhaye, E., Didierjean, C. \& Morel-Rouhier, M. (2015). Fungal. Genet. Biol., 83, 103.

Keywords: Cynaobacteria, Enzyme, Glutatione transferase, Glutathione, Methylglyoxal. 